Research Article

\title{
Optimal State Risk Scheduling Based on Selective Maintenance Strategy
}

\author{
Xingquan Ji, ${ }^{1}$ Xuan Zhang $\mathbb{D}^{1},{ }^{1}$ Yumin Zhang $\mathbb{D}^{1}{ }^{1}$ Xueshan Han, ${ }^{2}$ Ziyang Yin, ${ }^{1}$ \\ and Wei Wang ${ }^{3}$ \\ ${ }^{1}$ College of Electrical Engineering and Automation, Shandong University of Science and Technology, Qingdao 266590, China \\ ${ }^{2}$ Key Laboratory of Power System Intelligent Dispatch and Control, Shandong University, Jinan, Shandong 250061, China \\ ${ }^{3}$ College of Intelligent Equipment, Shandong University of Science and Technology, Tai'an, Shandong 271000, China
}

Correspondence should be addressed to Yumin Zhang; ymzhang2019@sdust.edu.cn

Received 21 March 2021; Revised 16 April 2021; Accepted 2 June 2021; Published 19 June 2021

Academic Editor: Giuseppe D'Aniello

Copyright ( $\odot 2021$ Xingquan Ji et al. This is an open access article distributed under the Creative Commons Attribution License, which permits unrestricted use, distribution, and reproduction in any medium, provided the original work is properly cited.

Because stochastic fault cases (i.e., opportunistic maintenance strategy) of the equipment are not considered in the conditionbased maintenance decision of the system, which will deviate from the actual situation, a system condition-based maintenance scheduling model considering opportunistic maintenance strategy is proposed in this paper. To implement the system maintenance strategies, the correlation set is formulated by considering the relationship among different equipment. According to renewal process theory, the availability of the correlation set considering planned maintenance and opportunity maintenance is deduced and the maintenance strategy of the system is realized. Then, to reflect the relationship between equipment maintenance and system operation, a system state scheduling model aiming at minimizing the sum of maintenance risk and failure risk as well as considering system resource constraints is proposed, thus obtaining the optimal maintenance schedule based on system state. Finally, a simple test system and IEEE-RTS79 node system are employed to demonstrate the feasibility and effectiveness of the proposed maintenance model, and it also verified that the proposed model can be integrated into the power system conditionbased maintenance theory.

\section{Introduction}

The development of equipment condition-based maintenance $(\mathrm{CBM})$ technology plays a positive role in improving the reliability and economy of system operation. And to trade off the contradictions and conflicts between equipment maintenance cost and system operation cost is a crucial issue in the research of equipment maintenance strategies $[1,2]$.

The purpose of maintenance activity is to extend equipment lifetime and/or reduce the probability of equipment failure [3]. The existing maintenance strategies for equipment can be broadly classified into corrective maintenance (CM) and preventive maintenance (PM) [4]. The former only performs maintenance activity after equipment fails. The latter can perform a maintenance plan of equipment before a failure occurs. The state-of-the-art in PM offers at least three basic approaches: (1) time-based maintenance, (2) condition-based maintenance (CBM), and (3) reliability-centered maintenance [5], in which the CBM approach determines maintenance decisions based on the deterioration state information. This information is provided by visual inspection, condition-monitoring technology, or online condition-monitoring system that can continuously monitor each equipment. The maintenance mechanism of the CBM has better performance because it develops a targeted maintenance plan for each equipment [6].

The conventional CBM methods described in the preceding literature focus on single equipment. That is to say, the maintenance policies are made for individual equipment components, rather than the whole system. However, for the power system, it is a typical multiequipment system in which the operation and maintenance policies are highly interdependent. The dependence between equipment within a 
complex system can be categorized into three groups: structural dependence, economic dependence, and stochastic dependence [7]. Therefore, the individual performance of equipment and the correlation between types of equipment need to be considered, when equipment maintenance strategy is formulated, and for a power system, the differences of equipment maintenance schedule also must be properly handled [8-10]. In [11], the concepts and models of maintenance risk and failure risk for the general series system were proposed, which fully consider the functional dependence, economic dependence, and stochastic dependence between equipment, and presented CBM optimization strategy; this $\mathrm{CBM}$ research has made significant progress. The study in [12] proposed the basic concept and mathematical model of CBM for power transmission system [13], which emphasized the relationship among equipment and the correlation and the coupling relation between system performance and demand.

The analytical method of multiequipment maintenance scheduling can be classified into two types: group maintenance $[8,9]$ and opportunistic maintenance $[10,14]$. In group maintenance, the maintenance actions or equipment to be replaced or repaired are grouped and one equipment in a group fails; the whole group is maintained or replaced. In opportunistic maintenance, the PM and CM activities are combined when certain economic and/or technical criteria are satisfied. In other words, some PM actions are performed when the system stops due to a failure $[15,16]$. In both approaches, the maintenance schedule is based on already occurred failure or maintenance. Therefore, considering the equipment degradation and dependencies, it is important that schedule maintenance is formulated when the system is upped.

In CBM, the maintenance scheduling of equipment only viewed the stochastic failure as risk [17]. And to treat the emergency accident scene is not considered, which can inevitably affect the goal of the maintenance decision. It shows that there are few researches about CDM strategy of equipment. Therefore, stochastic failure is needed for indepth research. Conventionally, opportunistic maintenance is a common strategy, which generally relies on a combination with other maintenance strategies (i.e., preventive maintenance) [18-22]. In other words, some preventive maintenance actions are performed after the system or equipment stops due to a failure, and other pieces of equipment can be also repaired together. By this way, the fixed maintenance cost and power outage loss can be shared, and the maintenance times of equipment can be reduced; thereby, the reliability and economy of system operation can be improved [13].

Visibly, opportunistic maintenance involves two types of maintenance modes according to different repair time: CM and PM [23]. For a long time, in the maintenance schedule decisions of multiequipment systems, the researchers on the opportunistic maintenance focused on the combination with the PM strategies of different equipment [24]. However, the treatment of the equipment stochastic failure case and the decision of the predetermined equipment CBM strategy are still separation, which will make a conservative or aggressive decision. How to integrate the two CM and PM organically for optimization decision is the core of this paper. Meanwhile, considering the opportunity maintenance in the CBM strategy of the system, there must exist the series logical relationship among the corresponding equipment.

Therefore, in this paper, the correlation set is defined and formulated to describe the correlation relationship among different equipment in multiequipment systems. Meanwhile, the theory of the renewal process is employed to deduce the availability of the correlation set considering the opportunistic maintenance policy. Based on the correlation set, the mathematical model of system maintenance risk and failure risk is deduced; we present a system maintenance scheduling model, which aims to minimize the sum of maintenance risk and failure risk while taking system resource constraint, to derive the optimal system condition-based maintenance scheduling. The case study results illustrate the feasibility and validity of the proposed maintenance model, and this model can be integrated into the theory of CBM for power systems, which will have a prospect of practical engineering application.

The major contributions of this paper are summarized as follows:

(1) The conception of the correlation set is proposed to describe the relationship among different equipment. Then taking the correlation set as the basic unit, a system CBM decision-making model based on correlation set decomposition is proposed, which can effectively reduce the maintenance cost caused by the scheduled maintenance activities of the system in multiequipment system.

(2) Based on the renewal process theory, the analytical expressions of equipment risk and system operation risk considering opportunity maintenance are derived, and the mathematical model of system CBM decision-making is reformulated. The opportunity maintenance strategy can not only express the risk level of system operation more accurately but also improve the reliability and economy of power system operation.

(3) A selective opportunistic maintenance strategy is proposed, and a quantitative method of equipment risk and system operation risk based on selective opportunity maintenance strategy considering the system maintenance time constraints and resource constraints is proposed, which not only enhances the integration between equipment $\mathrm{CBM}$ and system operation decision-making but also further improves the reliability and economy of the system operation. 
The rest of this paper is organized as follows. Section 2 introduces the formulation of the correlation set. Section 3 introduces the availability of the correlation set based on opportunistic maintenance. Section 4 defines the risk of system maintenance and the risk of failure. Section 5 presents the mathematical model of system state overhaul decision. Section 6 gives the solution of the model. The numerical results are provided in Section 7, and the conclusions are drawn in Section 8.

\section{Formulation of Correlation Set}

Correlation set is defined as the collection of equipment satisfying the following relevance during the system operation. Any equipment maintenance or failure inside the correlation set will cause outages of all equipment within this correlation set. The introduction of the correlation set is shown in Figure 1, where equipment $\mathrm{T} 1$ and equipment $\mathrm{B} 1$ constitute a correlation set. According to the actuating logic of relay protection [25-28], the failure of any equipment in the correlation set (e.g., an active equipment failure occurs) will cause the whole correlation set to stop operating after the switching operation (fault removal and fault recovery). Since the failure of the correlation set will cause some economic losses, considering the correlation relation between equipment, the opportunistic maintenance on other equipment can be used to carry out during maintenance the failure of the correlation set. In addition, due to the use of automatic switching operation equipment, the duration of equipment switching operation is shorter than the duration of equipment maintenance. Therefore, opportunistic maintenance during equipment switching operation is not considered in this paper.

For the primary equipment [29] in the system (bus, transformer, generator, circuit, and breaker), the decisionmaking principle of the correlation set is given.

(1) Enumerate the single failure of each primary equipment $i$ in the system, and consider the actions of the relay protection device, so as to determine the equipment set that the system finally exits operation after the switching operation, and it is marked as $A G_{i}$, thus establishing the mapping relationship between equipment $i$ and the outage set.

(2) Select any equipment $i$.

(3) If there is only the equipment $i$ in the set $A G_{i}$, then the equipment is a correlation set. Otherwise, for any equipment $j\left(j \in A G_{i}\right)$ in the set $A G_{i}$, if it simultaneously satisfies $i \in A G_{j}$, it can be determined that equipment $j$ and equipment $i$ belong to the same correlation set. After completing the ergodic process for all the internal equipment, the correlation set to which the equipment $i$ belongs and its composition can be determined.

After completing the ergodic process for all equipment of the system in accordance with the above decision-making principles, the system can be decomposed into correlation set and then take the correlation set as a unit to make maintenance decisions.

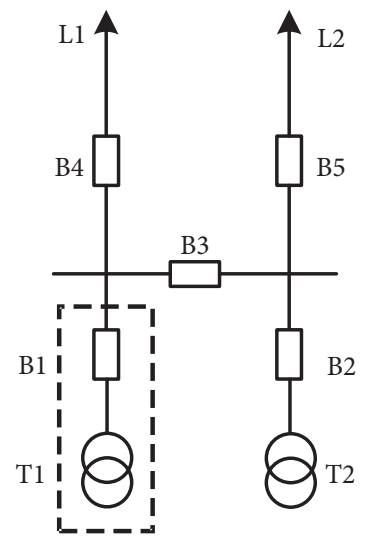

FIgURE 1: Demonstration of correlation.

\section{Availability of the Correlation Set Based on Opportunistic Maintenance}

During the research period, the equipment to be overseen in the system is obtained according to the equipment condition-monitoring and prediction technology, and on this basis, the correlation set that needs to be scheduled for planned outage is determined. However, the opportunistic maintenance inside the correlation set makes a series number of combinations, the impacts of different combinations on the system operation are variable. To maximize the operation performance of the system, it is necessary to comprehensively consider the actual combination state and system operation condition. Thus, the optimal opportunistic maintenance strategy can be determined through the analysis of the various combinations of equipment.

To make it clear, we take the simultaneously scheduled maintenance of equipment within the correlation set as an example to solve the availability expression of the correlation set corresponding to different opportunistic maintenance strategies. In other cases, the derivation method can be deduced in a similar way.

The derivation is based on the following three assumptions:

(1) The correlation set is composed of $n$ equipment, and individual equipment is mutually independent. The first life expectancy of equipment $i$ is subject to exponential distribution $F_{i}(t)=1-e^{-\lambda_{i, 1} t}$, and the repair time of equipment $i$ is subject to exponential distribution $G_{i}(t)=1-e^{-\widetilde{\mu}_{i} t}, \forall i=1,2, \ldots, n$.

(2) During the research periods, the failure rate of equipment has dropped after the first maintenance, and the decline relates to the adopted maintenance strategy (such as full complete overhaul, incomplete maintenance strategy [30]). To simplify the problem analysis, it is assumed that the failure rate of equipment $i$ decreases to $\lambda_{i, 0}$. Then the failure rate of equipment $i$ is considered constant within the research periods of maintenance scheduling. Thereby, the two-state transition process of the equipment is shown in Figure 2. 
(3) After the first maintenance, $A_{i, 0}(t)$ and $A_{i, 1}(t)$ are the availability functions of equipment $i$ when it is in the initial working state and fault state, respectively. The mathematical expression can be expressed as [31]

$$
\left\{\begin{array}{l}
A_{i, 0}(t)=\frac{\mu_{i}}{\lambda_{i, 0}+\mu_{i}}+\frac{\lambda_{i, 0}}{\lambda_{i, 0}+\mu_{i}} e^{-\left(\lambda_{i, 0}+\mu_{i}\right) t}, \\
A_{i, 1}(t)=\frac{\mu_{i}}{\lambda_{i, 0}+\mu_{i}}-\frac{\mu_{i}}{\lambda_{i, 0}+\mu_{i}} e^{-\left(\lambda_{i, 0}+\mu_{i}\right) t},
\end{array}\right.
$$

where " 0 " indicates working state, " 1 " indicates fault state, $\mu_{i}$ is the failure repair rate of equipment $i(i=1,2$, $\ldots, n), t_{m}$ is the start time of maintenance schedule of the correlation set, and $d_{i}$ is the maintenance duration of equipment $i(i=1,2, \ldots, n)$. Because the equipment in the correlation set is scheduled for maintenance at the same time, the maintenance time of the correlation set is $d_{m}=\max \left(d_{1}, d_{2}, \ldots, d_{n}\right)$. The first fault state transition process of correlation set is shown in Figure 3 , and the individual state is defined as follows:

State 0: $n$ equipment is all working, and the correlation set is in the working state.

State $i(i=1,2, \ldots, n)$ : equipment $i$ is failure, and the correlation set is in the outage state.

As shown in Figure 3, the following equation set can be derived according to the Markov state equation [32]:

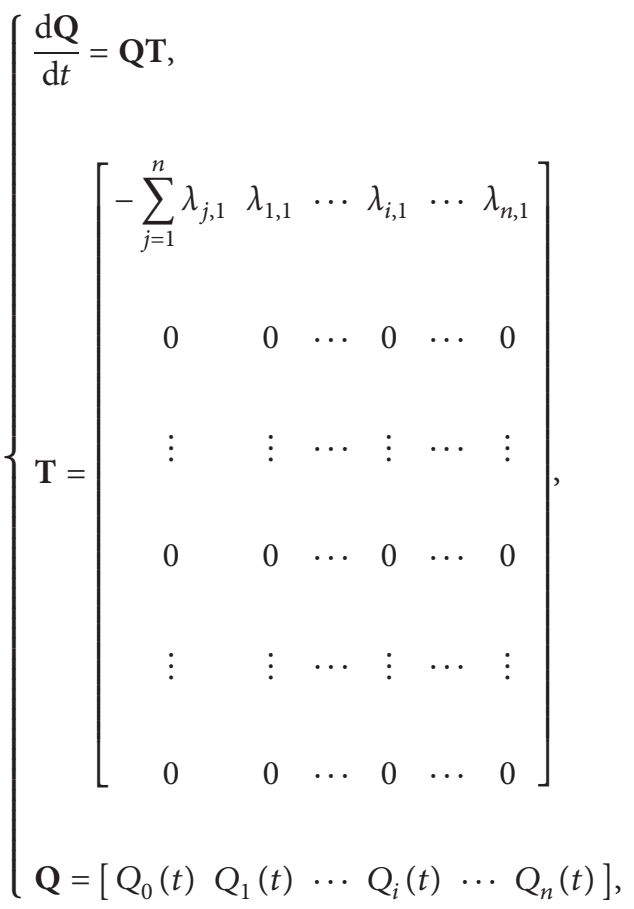

where $Q_{i}(t)$ is the probability of the correlation set when it is in the state $i$ at period $t$.

According to (2), the probability distribution of the correlation set state during the research periods can be obtained. Thereby, the availability expressions of the correlation set corresponding $n+1$ opportunistic maintenance strategy can be derived.

3.1. The Correlation Set Is in the Working State before the Schedule Maintenance. $Q_{0}(t)$ is the probability of the correlation set when it is in the working state at any time $t$ that belongs to the interval $\left[0, t_{m}\right]$, and $Q_{0}\left(t_{m}\right)$ is the implementation probability of the correlation set maintenance as scheduled. The availability of the correlation set after the completion of the scheduled maintenance can be obtained according to (1).

$$
A_{\text {case }, 0}(t)= \begin{cases}Q_{0}(t), & t<t_{m}, \\ 0, & t_{m} \leq t<t_{m}+d_{m}, \\ Q_{0}\left(t_{m}\right) \prod_{i=1}^{n} A_{i, 0}\left(t-t_{m}-d_{i}\right), & t \geq t_{m}+d_{m} .\end{cases}
$$

3.2. The Correlation Set Is in the Fault State before Schedule Maintenance. Since the correlation set is composed of $n$ equipment, according to the causes that caused the outage of the correlation set, there are $n$ possible cases that should be considered.

Case $i(i=1,2, \ldots, n)$ indicates that the failure of equipment $i$ causes outage of the correlation set. When it happens, the equipment $i$ will be overhauled, and then the state of equipment $i$ is improved. Meanwhile, the scheduled maintenance of equipment $i$ will be canceled according to [33]. It should be pointed out that, in practice, the failure rate may rise instead of decreasing because of the inadequate equipment maintenance (such as maintenance personnel's misoperation). However, this situation has a low probability [26]. Therefore, to highlight the idea of our work, the influence on maintenance decision will not be considered, even if the proposed model in this paper is applicable to this situation.

Since the failure of equipment $i$ causes outage of the correlation set, the availability expressions of the correlation set can be obtained according to whether to perform opportunistic maintenance for the remaining $n-1$ equipment. Therefore, for the remaining $n-1$ equipment, there are $2^{n-1}$ combinations of opportunistic maintenance, and the availability expressions of the correlation set for the different combinations can be calculated by 


$$
\begin{aligned}
& A_{\text {case }, i}(t)= \begin{cases}\int_{0}^{t} A_{i, 1}(t-u) \prod_{j \in \Omega_{i}^{1}} A_{j, 0}\left(t-u-d_{j}\right) \varepsilon\left(t-u-d_{j}\right) \cdot \prod_{k \in \Omega_{i}^{2}} A_{k, d e}(t-u) \mathrm{d} Q_{i}(u), & t<t_{m}, \\
\int_{0}^{t_{m}} A_{i, 1}(t-u) \prod_{j \in \Omega_{i}^{1}} A_{j, 0}\left(t-u-d_{j}\right) \varepsilon\left(t-u-d_{j}\right) \cdot \prod_{k \in \Omega_{i}^{2}} \widetilde{A}_{k}(t-u) \mathrm{d} Q_{i}(u), & t \geq t_{m},\end{cases} \\
& A_{k, d e}(t)=1-F_{k}(t)+\int_{0}^{t} A_{k, 0}(t-u) \mathrm{d} W_{k}(u) \\
& W_{k}(t)=\int_{0}^{t} G_{k}(t-u) \mathrm{d} F_{k}(u),
\end{aligned}
$$

where $\Omega_{i}^{1}$ and $\Omega_{i}^{2}$ are the set of equipment in which opportunistic maintenance is carried out and not carried out inside the correlation set after equipment $i$ failure, respectively, and there are $2^{n-1}$ possible values. $\varepsilon(t)$ is the unit step function. $A_{k, d e}(t)$ is the availability function of equipment $k$ when the scheduled maintenance is not considered and meets the delayed renewal process [33]. $W_{k}(t)$ is the periodic probability distribution function of equipment $k$ during the first update period. $\widetilde{A}_{k}(t-u)$ represents the availability of equipment $k\left(k \in \Omega_{i}^{2}\right)$ at the period $t\left(t \geq t_{m}\right)$ when equipment $i$ makes failure at the period $u\left(u \leq t_{m}\right)$. The specific derivation process of $\widetilde{A}_{k}(t-u)$ is given.
The stochastic process $\left\{X_{k}(t), t \geq 0\right\}$ is introduced to indicate the state changing process of equipment $k$ and satisfies

$$
X_{k}(t)= \begin{cases}0, & \text { if equipment } k \text { is in working state at period } t, \\ 1, & \text { if equipment } k \text { is in fault state at period } t,\end{cases}
$$

where $X_{k}(u)=0$ is given and $\xi_{k}$ is the time before equipment $k$ first failure when it is in working state at the period $u$ $\left(u \leq t_{m}\right)$. Then, $\widetilde{A}_{k}(t-u)$ can be expressed by full probability equation [34].

$$
\tilde{A}_{k}(t-u)=P\left\{\xi_{k}>t_{m}-u, X_{k}(t)=0 \mid X_{k}(u)=0\right\}+P\left\{\xi_{k} \leq t_{m}-u, X_{k}(t)=0 \mid X_{k}(u)=0\right\} .
$$

The first term of the right side in (8) represents equipment $k$ working reliably to the time $t_{m}$, the maintenance of the equipment $k$ is carried out according to the schedule, and the value of this item is

$$
\begin{array}{r}
P\left\{\xi_{k}>t_{m}-u, X_{k}(t)=0 \mid X_{k}(u)=0\right\} \\
= \begin{cases}0, & t_{m} \leq t<t_{m}+d_{k}, \\
{\left[1-F_{k}\left(t_{m}-u\right)\right] A_{k, 0}\left(t-t_{m}-d_{k}\right),} & t \geq t_{m}+d_{k} .\end{cases}
\end{array}
$$

The second term of the right side in (8) indicates equipment $k$ malfunctions before the period $t_{m}$, the equipment maintenance plan is transformed into postfault maintenance, and then

$$
\begin{array}{r}
P\left\{\xi_{k} \leq t_{m}-u, X_{k}(t)=0 \mid X_{k}(u)=0\right\} \\
=\int_{0}^{t_{m}-u} P\left\{X_{k}(t)=0 \mid X_{k}(u)=0, \xi_{k}=y\right\} \mathrm{d} P\left\{\xi_{k} \leq y\right\}=\int_{0}^{t_{m}-u} A_{k, 0}(t-u-y) \mathrm{d} F_{k}(y) .
\end{array}
$$

Considering the above $n+1$ cases, the availability of the correlation set corresponding to the different opportunistic maintenance strategies can be obtained by

$$
A(t)=\sum_{i=0}^{n} A_{\text {case }, i}(t)
$$

where (11) is the availability expression of the correlation set in the first failure. Similarly, it can be extended to the case where there is multiple opportunistic maintenance in the correlation set. Visibly, if the opportunistic maintenance is considered, there will be many combinations of opportunistic maintenance of equipment in 
the correlation set. Otherwise, it is a traditional maintenance decision-making problem according to [35].

\section{The Risk of System Maintenance and the Risk of Failure}

4.1. Equipment Maintenance Risk and Failure Risk. Equipment maintenance risk and failure risk are the individual equipment losses which are caused by the equipment maintenance (schedule maintenance or opportunistic maintenance) and malfunction, respectively. Taking the correlation set as a unit, the maintenance risk and failure risk of the equipment were calculated by considering the abovementioned $n+1$ cases.

In this paper, it is assumed that $N_{i, j}(t)$ represents the fault number of the equipment $i$ which is under the condition of $j(j \in\{0,1\})$ and belongs to $(0, t]$ after the first maintenance. We have

$$
m_{i, j}(t)=E\left\{N_{i, j}(t)\right\}
$$

where $E\{\cdot\}$ represents the mathematical expectation of the random variable $\cdot$

According to [34], we have

$$
\left\{\begin{array}{l}
m_{i, 0}(t)=\frac{\lambda_{i, 0} \mu_{i}}{\lambda_{i, 0}+\mu_{i}} t+\frac{\lambda_{i, 0}^{2}}{\left(\lambda_{i, 0}+\mu_{i}\right)^{2}}\left[1-e^{-\left(\lambda_{i, 0}+\mu_{i}\right) t}\right], \\
m_{i, 1}(t)=\frac{\lambda_{i, 0} \mu_{i}}{\lambda_{i, 0}+\mu_{i}} t-\frac{\lambda_{i, 0} \mu_{i}}{\left(\lambda_{i, 0}+\mu_{i}\right)^{2}}\left[1-e^{-\left(\lambda_{i, 0}+\mu_{i}\right) t}\right] .
\end{array}\right.
$$

The equipment maintenance risk and failure risk are deduced by considering the following $n+1$ cases:

(1) If the correlation set reliably runs to the scheduled maintenance time $t_{m}$, then the equipment maintenance risk is the individual loss of equipment caused by scheduled maintenance, which can be expressed as

$$
C R_{\text {case }, 0}^{P}=Q_{0}\left(t_{m}\right) \sum_{i=1}^{n} C_{P, i},
$$

where $C_{P, i}$ is the needed maintenance cost of equipment $i$.

When this event occurs, the equipment failure risk is the individual equipment loss caused by the equipment failure; it can be expressed as

$$
C R_{\text {case }, 0}^{F}=Q_{0}\left(t_{m}\right) \sum_{i=1}^{n} m_{i, 0}\left(T-t_{m}-d_{i}\right) C_{F, i},
$$

where $C_{F, i}$ is the maintenance cost of the equipment $i$ which has been on the fault state and $T$ is the number of the research periods.

(2) If the correlation set cannot run reliably to the scheduled maintenance time $t_{m}$, the following $n$ cases should be considered according to the different equipment causing the outage of association set.

For case $i(i=1,2, \ldots, n)$, the failure of equipment $i$ causes the outage of correlation set. Meanwhile, the equipment maintenance risk and the failure risk can be calculated according to whether or not to carry out the opportunistic maintenance for the remaining $n-1$ equipment.

The equipment maintenance risk can be expressed as

$$
C R_{\text {case }, i}^{P}=Q_{i}\left(t_{m}\right) \sum_{j \in \Omega_{i}^{1}} C_{P, j}+\int_{0}^{t_{m}} \sum_{k \in \Omega_{i}^{2}}\left[1-F_{k}\left(t_{m}-u\right)\right] C_{P, k} \mathrm{~d} Q_{i}(u),
$$

where the first term on the right (16) indicates that the individual losses are caused by opportunistic maintenance, the second item indicates the individual losses of equipment due to schedule maintenance. The equipment failure risk can be expressed as

$$
\begin{aligned}
C R_{\text {case }, i}^{F} & =\int_{0}^{t_{m}}\left[1+m_{i, 1}(T-u)\right] C_{F, i} \mathrm{~d} Q_{i}(u)+\int_{0}^{t_{m}} \sum_{j \in \Omega_{i}^{1}} m_{j, 0}\left(T-u-d_{j}\right) C_{F, j} \mathrm{~d} Q_{i}(u)+\int_{0}^{t_{m}} \sum_{k \in \Omega_{i}^{2}} n_{k}(u, T) C_{F ; k} \mathrm{~d} Q_{i}(u), \\
n_{k}(u, T) & =E\left\{N_{k, 0}(T) \mid \xi_{k}>t_{m}-u\right\} P\left\{\xi_{k}>t_{m}-u\right\}+\int_{0}^{t_{m}-u} E\left\{N_{k, 0}(T) \mid \xi_{k}=y\right\} \mathrm{d} P\left\{\xi_{k} \leq y\right\} \\
& =m_{k, 0}\left(T-t_{m}-d_{k}\right)\left[1-F_{k}\left(t_{m}-u\right)\right]+\int_{0}^{t_{m}-u}\left[1+m_{k, 1}(T-u-y)\right] \mathrm{d} F_{k}(y),
\end{aligned}
$$

where $n_{k}(u, T)$ is the average fault number of equipment $k$ during the research period when equipment $i$ malfunctions at the period $u\left(u \leq t_{m}\right)$. When $x_{k}(u)=0, n_{k}(u, T)$ can be expressed by the total probability.

Considering the above $n+1$ cases, the equipment maintenance risk and the failure risk of a correlation set can be expressed as

$$
R_{\text {Comp }}=\sum_{i=0}^{n}\left(C R_{\text {case }, i}^{P}+C R_{\text {case }, i}^{F}\right)
$$

4.2. Power System Operation Risk. System operation risk refers to the maintenance risk and failure risk of the system caused by equipment maintenance failure during the 


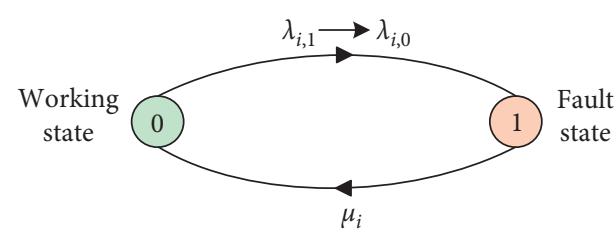

Figure 2: Transition process of the equipment state.

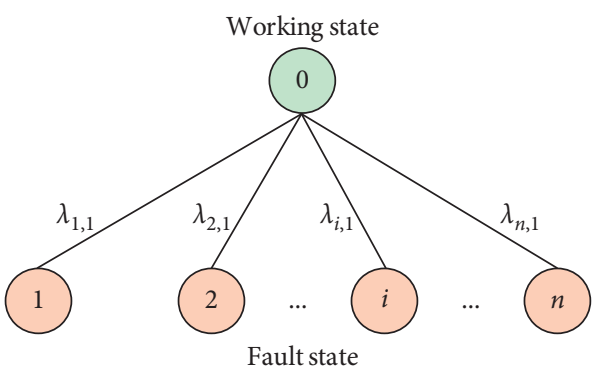

Figure 3: The first fault state transition process of correlation set.

research periods. The expected energy not supplied (EENS) is adopted to describe the risk loss of the system that includes the scheduled and unscheduled loss of load.

$$
\begin{aligned}
R_{\text {Sys }} & =\sum_{t=1}^{T} \sum_{s \in S(t)} P_{s}(t)\left[\operatorname{sev}_{s, 1}(t)+\operatorname{sev}_{s, 2}(t)\right] \\
P_{s}(t) & =\prod_{i=1}^{N-N_{s}} A_{i}(t) \prod_{j=1}^{N_{s}}\left(1-A_{j}(t)\right) \\
\operatorname{sev}_{s, 1}(t) & =\sum_{i \in N_{d}} \min \left(L_{s, i}(t), L_{0, i}(t)\right) c_{p} \\
\operatorname{sev}_{s, 2}(t) & =\sum_{i \in N_{d}} \max \left(0, L_{s, i}(t)-L_{0, i}(t)\right) c_{f}
\end{aligned}
$$

where $S(t)$ is the accident set of the system at the period $t$, which can be determined by analysis $N-1$ for complex power systems. $P_{s}(t)$ is the probability of the accident $s$ occurred at the period $t . \operatorname{sev}_{s, 1}(t)$ and $\operatorname{sev}_{s, 2}(t)$ are the loss of scheduled load and unscheduled load at the period $t$, respectively. $N$ is the number of the correlation set decomposed by system. $N-N_{s}$ and $N_{s}$ are the number of the correlation set in available state and unavailable state in accident $s$, respectively. $N_{d}$ is the system load set. $L_{0, i}(t)$ is the scheduled load loss of node $i$ at the period $t$, which can be determined by the system state of the schedule maintenance failure of equipment. $L_{s, i}(t)$ is the load loss of node $i$ corresponding to the accident $s$ at the period $t$. For different systems (such as substation), relevant algorithms have been very mature, and the detailed content can be found in [32]. $c_{p}$ and $c_{f}$ are the scheduled and unscheduled load unit loss, respectively $\left(c_{p}<c_{f}\right)$.

\section{The Mathematical Model of System State Overhaul Decision}

During the research period, to minimize the sum of equipment maintenance risk, failure risk, and system operation risk, the optimal decision-making model of system $\mathrm{CBM}$ is constructed, as shown in

$$
\min \sum_{i=1}^{N} R_{i, \text { Comp }}+R_{\text {Sys }}
$$

The system maintenance decision should meet the following constraints.

5.1. Maintenance Time Constraints. The scheduled maintenance of the equipment should be arranged within a given time interval.

$$
\begin{cases}x_{i}(t)=0, & t<b_{i} \text { or } t>e_{i} \\ x_{i}(t)=1, & b_{i} \leq t \leq e_{i}\end{cases}
$$

where $x_{i}(t)$ represents the state of equipment $i$ at period $t$, if the maintenance of the equipment $i$ in period $t$ is serviced; $x_{i}(t)=1$; otherwise, $x_{i}(t)=0 . b_{i}$ and $e_{i}$ are, respectively, the earliest period and the latest period when the equipment $i$ is allowed to be overhauled.

\subsection{Maintenance Resource Constraints.}

$$
\sum_{i=1}^{N_{M}} x_{i}(t) r_{i, k} \leq I_{k}(t), \quad \forall k, t,
$$

where $N_{M}$ is the number of equipment waiting for maintenance in the system. $r_{i, k}$ is the maintenance demand of equipment $i$ for resource $k . I_{k}(t)$ is the availability of resource $k$ at period $t$.

5.3. Synchronous Maintenance Constraints. For the equipment $i$ and $j$ to be overhauled in the same correlation set, they should be arranged to be overhauled together to avoid repeated power outages

$$
x_{i}(t)=x_{j}(t), \quad \forall t .
$$

\section{The Solution of Model}

The decision-making of system maintenance is a combinatorial optimization problem with a variety of constraints. Therefore, the traditional mathematical optimization method may be difficult to solve the problem, it is available to adopt intelligent search technique, and the genetic algorithms are used in this paper. The detailed solving process is as follows:

Step 1: making the correlation set of the system.

Step 2: population initialization. Each individual in the population represents a maintenance scheme, which corresponds to the maintenance time of the equipment to be overhauled in the correlation set and the opportunity maintenance strategy will be implemented.

Step 3: for each individual of the population, we should judge whether it meets the constraints of the system maintenance decision-making. If it is satisfied, the sum of the system maintenance risk and failure risk corresponding to the maintenance scheme is calculated as 
the individual fitness value. Otherwise, a larger value is directly assigned as the corresponding fitness value.

Step 4: judge the convergence of the genetic algorithm. If the algorithm converges, the individual with the least fitness is used as the solution of the model. Otherwise, the individuals will cross over and mutate, regenerate a new population, and return Step 3.

\section{Example and Analysis}

7.1. Simple Structure of Substation. As shown in Figure 4, a simple substation structure [26-28] is used to reveal the mechanism of the proposed model in this paper. The proposed maintenance strategies are studied over 180 days and 2 days as a basic unit; therefore, it can be divided into 90 periods. The status information and related parameters of the equipment to be overhauled in the system are shown in Tables 1 and 2, respectively. It is assumed that the L1, L2, and L3 can operate reliably over the whole research periods, and the availability of other equipment is shown in Table 3. The system load curve in the research periods is shown in Figure 5. In this paper, the unit loss of the scheduled and unscheduled load loss is 0.53 thousand yuan/(MW $\cdot \mathrm{h})$ and 10.53 thousand yuan/(MW.h), respectively.

7.1.1. Maintenance Strategy Analysis of Substation A. For substation a, when the equipment maintenance schedule is predetermined, the mechanism of different opportunistic maintenance strategies is analyzed. It is assumed that the maintenance schedule is arranged in the system load trough (the starting period of the scheduled maintenance is 60). The following three kinds of maintenance schemes are analyzed:

Scheme 1: before scheduled maintenance, if any equipment makes failure in the equipment set which is to schedule the pending maintenance equipment, then the opportunistic maintenance for the other pending maintenance equipment will be served.

Scheme 2: before scheduled maintenance, only the opportunistic maintenance of equipment B1 is considered.

Scheme 3: it is analyzed without considering the opportunistic maintenance.

By analyzing the above three maintenance schemes, the corresponding to the availability curve of the system can be obtained, which is shown in Figure 6.

Figure 6 shows the following.

Comparing Schemes 2 with 3, the system availability of Scheme 2 is slightly higher. This is because the opportunistic maintenance for equipment B1 is performed after equipment T1 failure. As shown in Tables 1 and 2, compared with the average failure repair time of equipment $\mathrm{T} 1$, the maintenance time of equipment B1 is shorter. From the deduce process of availability, it can be seen that the opportunistic maintenance reduces the increment of unscheduled downtime in terms of probability. Meanwhile, the operation status of equipment B1 improved by opportunistic maintenance before scheduled maintenance. As a result, the maintenance schedule of equipment B1 is canceled, which reduces the probability of system-scheduled outage.

Comparing Scheme 1 with Scheme 2, the system availability of Scheme 1 is slightly lower before scheduled maintenance. This is because that Scheme 1 further takes advantage of the outage of equipment B1 to overhaul equipment $\mathrm{T} 1$ on the basis of Scheme 2 , and the maintenance time of equipment $\mathrm{T} 1$ is longer compared with the average failure repair time of equipment B1. Therefore, in view of probability, the unscheduled outage time of the system significantly increased before scheduled maintenance in Scheme 1, thus reducing the system availability. As shown in Figure 6, the system availability of Scheme 1 in the scheduled maintenance interval is slightly higher; this is because the maintenance probability of the equipment $\mathrm{T} 1$ before scheduled maintenance increased due to the opportunistic maintenance strategy, thereby reducing the probability of the system-scheduled outage.

The risk index of the system corresponding to the above three maintenance schemes is illustrated in Table 4.

Table 4 shows the following.

Compared with Scheme 3, both the equipment risk and the system operation risk of Scheme 2 are reduced; the main reason is that the equipment $\mathrm{B} 1$ has been overhauled before scheduled maintenance is conducted by opportunistic maintenance, which reduces the possibility of further failure of equipment B1 and the loss of equipment failure. Meanwhile, the system outage again caused by scheduled maintenance of equipment B1 can be avoided. The reduced loss of equipment failure is greater than the increased outage loss of system which implements the opportunistic maintenance.

Comparing Scheme 1 with Scheme 2, the equipment risk of Scheme 1 is reduced, but the system operation risk of Scheme 1 is increased, which shows that Scheme 1 is beneficial to reducing the equipment loss, but Scheme 1 has a great influence on the unscheduled outage time of the system, because of the weak structure of substation a, so the main contradiction of maintenance decision is how to reduce the system operation risk. Although Scheme 1 reduces the equipment failure loss and system-scheduled outage loss, the increment of system outage loss caused by the implementation of the opportunistic maintenance is much greater than the decrement of the above two losses, which eventually leads to the total risk of Scheme 1 which is large in all time intervals.

7.1.2. Maintenance Strategy Analysis of Substation B. For the above three maintenance schemes, one can analyze the impacts of different opportunistic maintenance strategies on substation $\mathrm{b}$. Table 5 demonstrates the system risk indicators corresponding to the above three maintenance schemes.

Table 5 shows the following.

Comparing Scheme 2 and Scheme 3, it is concluded that both the equipment risk and the system operation risk have been reduced, which is consistent with that in Section 7.1.1.

Comparing Scheme 1 with Scheme 2, the equipment risk of Scheme 1 is reduced, but the system operation risk of 

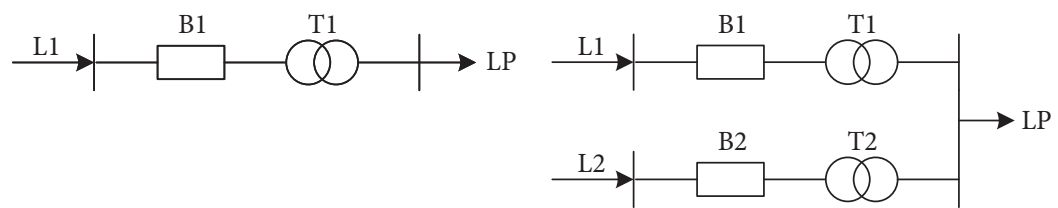

(a)

(b)

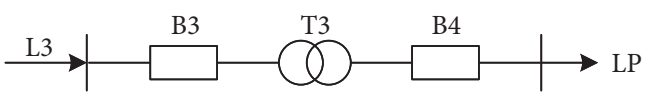

(c)

Figure 4: Substation configurations. (a) Substation a. (b) Substation b. (c) Substation c.

TABle 1: State of the equipment.

\begin{tabular}{lccc}
\hline Equipment & $\lambda_{1}$ (time/day) & $\lambda_{0}$ (time/day) & $\mu$ (time/day) \\
\hline T1 & 0.0035 & 0.001 & 0.042 \\
B1 & 0.0025 & 0.0005 & 0.11 \\
B3 & 0.003 & 0.0001 & 0.11 \\
B4 & 0.004 & 0.0003 & 0.11 \\
\hline
\end{tabular}

TABle 2: Maintenance data of the equipment.

\begin{tabular}{lccc}
\hline Equipment & $d$ (day) & $C_{P}\left(10^{4}\right.$ yuan $)$ & $C_{F}\left(10^{4}\right.$ yuan $)$ \\
\hline T1 & 14 & 20 & 90 \\
B1 & 6 & 5 & 10 \\
B3 & 6 & 5 & 10 \\
B4 & 6 & 5 & 10 \\
\hline
\end{tabular}

TABLE 3: Availability of substation equipment.

\begin{tabular}{lc}
\hline Equipment & Availability (\%) \\
\hline B2 & 99.8 \\
T2 & 99.8 \\
T3 & 99 \\
\hline
\end{tabular}

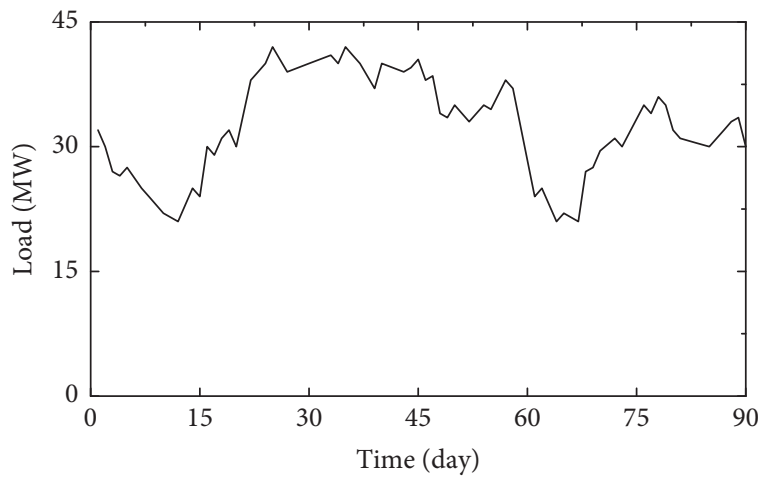

Figure 5: Load curve.

Scheme 1 is increased. However, due to the high reliability of the substation $b$, the proportion of system operating risk to the total system risk is small. At this time, how to reduce the risk of equipment becomes the main contradiction of maintenance decision, which leads to the decrement of equipment risk in Scheme 1 which is larger than the increment of system operation risk, and the total system risk of Scheme 1 is small in the whole research periods. It is also affected by the factors of individual performance of the equipment, opportunity of maintenance, the system load pattern, and so on.
Therefore, it is meaningful to take opportunistic maintenance strategy into system maintenance decisions, and a reasonable choice of equipment to implement the opportunistic maintenance strategy is also very important. Therefore, for all the time intervals, both the equipment risk and system operational risk should be considered to determine the opportunistic maintenance strategy of equipment from the point of systemic view.

7.1.3. Maintenance Strategy Analysis of Substation C. In view of substation $c$, when the equipment maintenance schedule is predesignated, the mechanism of different opportunity maintenance strategies is analyzed. This paper assumes that the initial maintenance period is 60. Then, analyze the following three maintenance schemes:

Scheme 1: before scheduled maintenance, if any equipment makes failure in the set of pending maintenance equipment, then the opportunistic maintenance for the other pending maintenance equipment will be served.

Scheme 2: before scheduled maintenance, only consider the opportunistic maintenance to the equipment B3.

Scheme 3: it is analyzed without considering the opportunistic maintenance. 


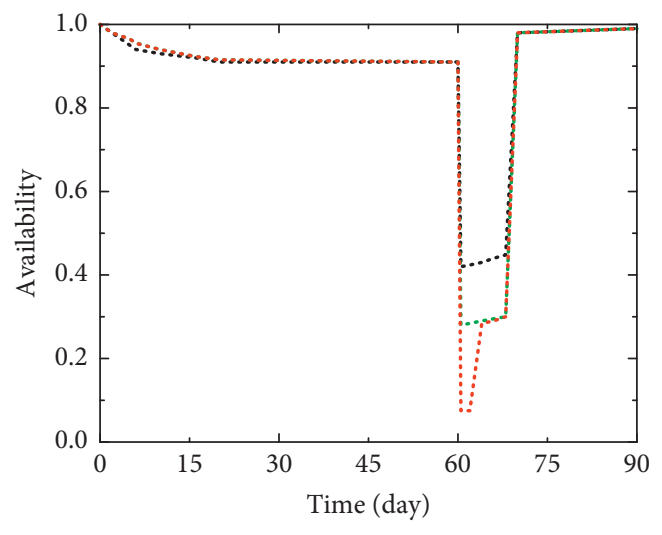

...... Scheme 1

...... Scheme 2

...... Scheme 3

Figure 6: Availability of the system.

TABLE 4: Maintenance strategy comparison of substation a.

\begin{tabular}{lccc}
\hline Scheme & Equipment risk & System operation risk & Total risk \\
\hline Scheme 1 & 54.33 & 10008.98 & 10063.31 \\
Scheme 2 & 56.25 & 9436.08 & 9492.33 \\
Scheme 3 & 56.39 & 9505.04 & 9561.43 \\
\hline
\end{tabular}

TABLE 5: Maintenance strategy comparison of substation b.

\begin{tabular}{lccc}
\hline Schemes & Equipment risk & System operation risk & Total risk \\
\hline Scheme 1 & 54.33 & 42.16 & 96.49 \\
Scheme 2 & 56.25 & 40.79 & 97.04 \\
Scheme 3 & 56.39 & 41.96 & 98.35 \\
\hline
\end{tabular}

TABLE 6: Maintenance strategy comparison of substation c.

\begin{tabular}{lccc}
\hline Schemes & Equipment risk & System operation risk & Total risk \\
\hline Scheme 1 & 13.20 & 6655.47 & 6668.67 \\
Scheme 2 & 13.44 & 6733.51 & 6746.95 \\
Scheme 3 & 13.72 & 6750.81 & 6764.53 \\
\hline
\end{tabular}

The risk index of the system corresponding to the above three maintenance schemes is illustrated in Table 6. The following conclusions can be obtained.

Comparing Scheme 2 with Scheme 3, both the equipment risk and the system operation risk have been reduced, which is due to the fact that the maintenance time of equipment B3 is shorter (compared to MTTR of equipment B4). Therefore, the opportunistic maintenance for equipment B3 will improve the operating condition before the scheduled maintenance, which is helpful to reduce the loss of equipment failure. Besides, from the perspective of system operation, the decrement of the system outage lost after opportunistic maintenance is greater than the system outage lost when implementing the opportunistic maintenance.

Scheme 1 is based on Scheme 2 and further implements opportunistic maintenance for equipment B4; it is easy to find that Scheme 1 can further reduce the equipment risk and operation risk, which is consistent with the results of Table 6. Therefore, the above examples fully show that this paper proposed system maintenance risk and failure risk, which can adequately quantify the impacts of different maintenance schemes on the individual performance of equipment and system operation risk, and the smaller the sum of system maintenance risk and failure risk is, the more advantageous to coordinate the relationship between the opportunistic maintenance of equipment and the system operation will be.

7.2. IEEE-RTS79. The IEEE-RTS79 system is adopted to further prove the effectiveness and significance of the proposed theoretical study, the system diagram is shown in Figure 7 . The research periods are 120 days, which is divided into 60 periods, and the duration of each period is 2 days. During the research periods, the scheduled maintenance is 


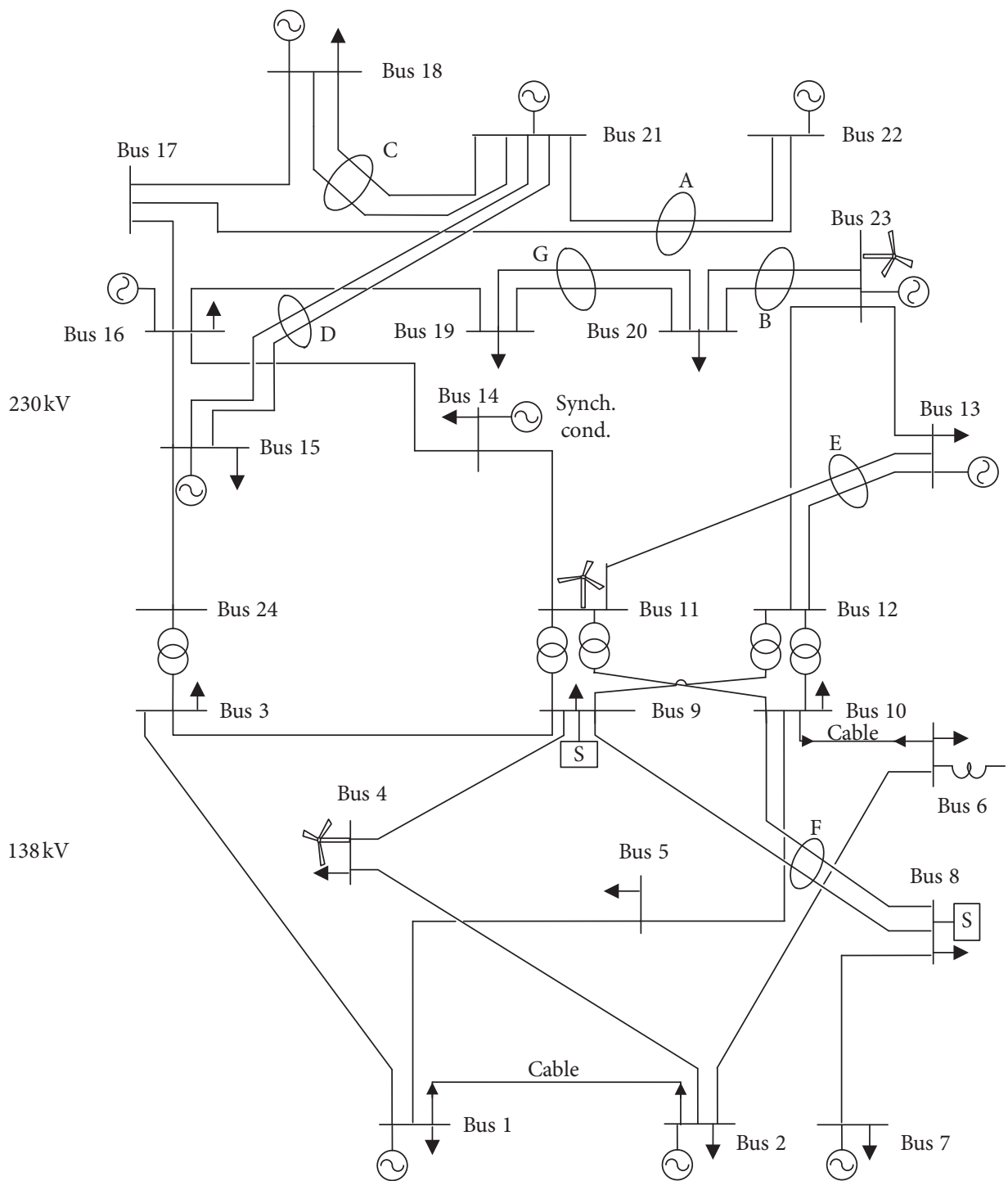

FIgURE 7: System diagram of IEEE-RTS79.

implemented on some equipment of bus 13 and bus 23 (as shown in Figure 8) and assumes that other types of equipment are all reliable operation. The status information and the maintenance parameters of equipment at each bus are shown in Tables 7 and 8 . The load data of each period of the system are shown in Table 9, and other detailed parameters can be found in [36]. The unit loss of scheduled and unscheduled load loss is 0.53 thousand yuan/MWh and 10.53 thousand yuan/MWh, respectively. In the process of system CBM decision, the load shedding of the system can be obtained by calculating the DC optimal power flow model.

Make decisions based on the following three maintenance schemes:

Scheme 1: before scheduled maintenance, if any equipment makes failures in the set of the pending maintenance equipment, then the opportunistic maintenance for the other pending maintenance equipment will be served.
Scheme 2: the selective opportunistic maintenance from the point of view of the system is implemented; it is the model proposed in this paper.

Scheme 3: it is analyzed without considering the opportunistic maintenance.

Table 10 is the correlation set decomposition results of the system. Tables 11 13 are the maintenance schedule, the optimal opportunistic strategy, and the risk of the IEEERTS79 system.

Tables 11 13 show the following:

(1) Contrasting the three maintenance schemes, the maintenance opportunity of equipment varies from the different maintenance schemes. Therefore, it demonstrates that the opportunistic maintenance has a great influence on the system operation and embodies the necessity of this study. 


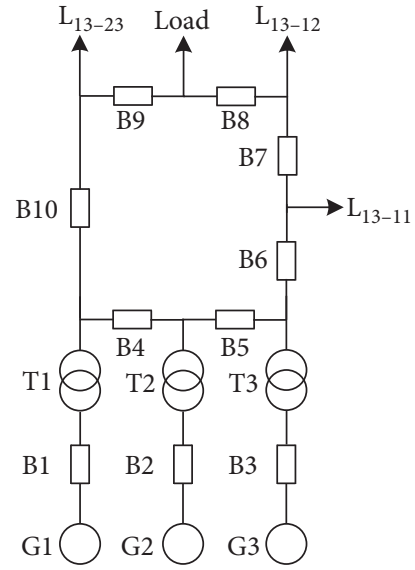

(a)

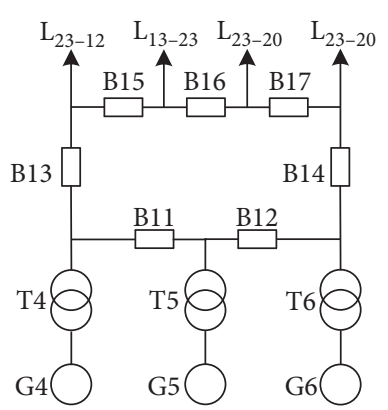

(b)

Figure 8: Electric connection scheme diagram. (a) Bus 13. (b) Bus 23.

TABLE 7: Maintenance data of the equipment in IEEE-RTS79 system.

\begin{tabular}{|c|c|c|c|c|c|c|}
\hline Equipment & $\lambda_{1}$ (time/day) & $\lambda_{0}$ (time/day) & $\mu$ (time/day) & $d$ (day) & $C_{p}\left(10^{4}\right.$ yuan $)$ & $C_{F}\left(10^{4}\right.$ yuan $)$ \\
\hline G2 & 0.0030 & 0.00075 & 0.0625 & 10 & 6 & 20 \\
\hline G3 & 0.0025 & 0.00075 & 0.0625 & 10 & 6 & 20 \\
\hline G4 & 0.0020 & 0.00075 & 0.0625 & 10 & 6 & 20 \\
\hline G6 & 0.0035 & 0.00075 & 0.0625 & 10 & 6 & 20 \\
\hline $\mathrm{T} 1$ & 0.0025 & 0.00050 & 0.0420 & 14 & 20 & 90 \\
\hline T3 & 0.0025 & 0.00050 & 0.0420 & 14 & 20 & 90 \\
\hline $\mathrm{T} 4$ & 0.0025 & 0.00050 & 0.0420 & 14 & 20 & 90 \\
\hline T6 & 0.0030 & 0.00050 & 0.0420 & 14 & 20 & 90 \\
\hline
\end{tabular}

TABLE 8: Equipment state of IEEE-RTS79 system.

\begin{tabular}{|c|c|c|c|c|}
\hline Equipment & $\lambda_{1}$ (time/day) & $\lambda_{0}$ (time/day) & $\mu$ (time/day) & $C_{p}\left(10^{4}\right.$ yuan $)$ \\
\hline G1 & 0.00285 & 0.00075 & 0.0625 & 20 \\
\hline G5 & 0.00350 & 0.00075 & 0.0625 & 20 \\
\hline $\mathrm{T} 2$ & 0.00285 & 0.00050 & 0.0420 & 90 \\
\hline T5 & 0.00300 & 0.00050 & 0.0420 & 90 \\
\hline B1 & 0.00285 & 0.00030 & 0.1100 & 10 \\
\hline B2 & 0.00400 & 0.00030 & 0.1100 & 10 \\
\hline B3 & 0.00350 & 0.00030 & 0.1100 & 10 \\
\hline B4 & 0.00070 & 0.00030 & 0.1100 & 10 \\
\hline B5 & 0.00090 & 0.00030 & 0.1100 & 10 \\
\hline B6 & 0.00100 & 0.00030 & 0.1100 & 10 \\
\hline B7 & 0.00100 & 0.00030 & 0.1100 & 10 \\
\hline B8 & 0.00100 & 0.00030 & 0.1100 & 10 \\
\hline B9 & 0.00100 & 0.00030 & 0.1100 & 10 \\
\hline B10 & 0.00100 & 0.00030 & 0.1100 & 10 \\
\hline B11 & 0.00100 & 0.00030 & 0.1100 & 10 \\
\hline B12 & 0.00100 & 0.00030 & 0.1100 & 10 \\
\hline B13 & 0.00090 & 0.00030 & 0.1100 & 10 \\
\hline B14 & 0.00080 & 0.00030 & 0.1100 & 10 \\
\hline B15 & 0.00100 & 0.00030 & 0.1100 & 10 \\
\hline B16 & 0.00800 & 0.00030 & 0.1100 & 10 \\
\hline B17 & 0.00800 & 0.00030 & 0.1100 & 10 \\
\hline
\end{tabular}


TABLE 9: Peak load in percent of annual peak in IEEE-RTS79 system.

\begin{tabular}{|c|c|}
\hline Time & Load \\
\hline 1 & 117.1 \\
\hline 2 & 116.9 \\
\hline 3 & 110.1 \\
\hline 4 & 101.9 \\
\hline 5 & 101.3 \\
\hline 6 & 102.8 \\
\hline 7 & 98.5 \\
\hline 8 & 94.4 \\
\hline 9 & 93.9 \\
\hline 10 & 89.3 \\
\hline 11 & 88.1 \\
\hline 12 & 87.6 \\
\hline 13 & 92.7 \\
\hline 14 & 96.8 \\
\hline 15 & 93.9 \\
\hline 16 & 93.9 \\
\hline 17 & 91.6 \\
\hline 18 & 96.2 \\
\hline 19 & 97.3 \\
\hline 20 & 95.1 \\
\hline 21 & 109.2 \\
\hline 22 & 110.1 \\
\hline 23 & 112.3 \\
\hline 24 & 114.6 \\
\hline 25 & 112.8 \\
\hline 26 & 118.6 \\
\hline 27 & 113.4 \\
\hline 28 & 112.3 \\
\hline 29 & 107.7 \\
\hline 30 & 98.5 \\
\hline 31 & 112.3 \\
\hline 32 & 110.1 \\
\hline 33 & 107.7 \\
\hline 34 & 104.2 \\
\hline 35 & 105.4 \\
\hline 36 & 113.4 \\
\hline 37 & 104.2 \\
\hline 38 & 98.5 \\
\hline 39 & 99.6 \\
\hline 40 & 90.4 \\
\hline 41 & 89.5 \\
\hline 42 & 94.6 \\
\hline 43 & 90.4 \\
\hline 44 & 87.6 \\
\hline 45 & 88.1 \\
\hline 46 & 90.4 \\
\hline 47 & 89.3 \\
\hline 48 & 92.7 \\
\hline 49 & 97.5 \\
\hline 50 & 95.7 \\
\hline 51 & 98.5 \\
\hline 52 & 106.8 \\
\hline 53 & 110.1 \\
\hline 54 & 114.3 \\
\hline 55 & 110.9 \\
\hline 56 & 118.1 \\
\hline 57 & 116.9 \\
\hline 58 & 116.1 \\
\hline 59 & 114.4 \\
\hline 60 & 113.1 \\
\hline
\end{tabular}

TABLE 10: Correlation set decomposition of bus 13 and 23 .

\begin{tabular}{lc}
\hline Correlation set & Internal equipment \\
\hline 1 & G1, B1, T1 \\
2 & G2, B2, T2 \\
3 & G3, B3, T3 \\
4 & B4 \\
5 & B5 \\
6 & B6 \\
7 & B7 \\
8 & B8 \\
9 & B9 \\
10 & B10 \\
11 & G4, T4 \\
12 & G5, T5 \\
13 & G6, T6 \\
14 & B11 \\
15 & B12 \\
16 & B13 \\
17 & B14 \\
18 & B15 \\
19 & B16 \\
20 & B17 \\
\hline
\end{tabular}

TABLE 11: Maintenance schedule of IEEE-RTS79 system.

\begin{tabular}{lccc}
\hline Equipment & \multicolumn{3}{c}{ Starting period of maintenance (2 days) } \\
& Scheme 1 & Scheme 2 & Scheme 3 \\
\hline G2 & 15 & 38 & 40 \\
G3 & 8 & 8 & 10 \\
G4 & 13 & 48 & 39 \\
G6 & 6 & 6 & 6 \\
T1 & 40 & 13 & 13 \\
T3 & 8 & 8 & 10 \\
T4 & 13 & 48 & 39 \\
T6 & 6 & 6 & 6 \\
\hline
\end{tabular}

TABLE 12: Optimal opportunistic strategy of IEEE-RTS79 system.

\begin{tabular}{lc}
\hline $\begin{array}{l}\text { Equipment of pending } \\
\text { maintenance }\end{array}$ & $\begin{array}{c}\text { Equipment of opportunistic } \\
\text { maintenance }\end{array}$ \\
\hline G2 & T2 \\
G3 & T3 \\
G4 & T4 \\
G6 & T6 \\
T1 & G1 \\
T3 & G3 \\
T4 & G4 \\
T6 & G6 \\
\hline
\end{tabular}

TABLE 13: Risk of IEEE-RTS79 system.

\begin{tabular}{lccc}
\hline Schemes & Equipment risk & System operation risk & Total risk \\
\hline Scheme 1 & 251.95 & 28956.92 & 29208.87 \\
Scheme 2 & 280.96 & 18003.65 & 18284.61 \\
Scheme 3 & 272.39 & 28346.83 & 28619.22 \\
\hline
\end{tabular}

(2) The different maintenance schemes have different effects on system operation. By contrasting Scheme 1 and Scheme 3, the system total risk of the corresponding maintenance strategy proposed model in 
this paper is the smallest, which effectively coordinates the relationship between equipment maintenance and system operation.

Therefore, in the practical application, the maintenance decision should be based on the proposed model and choose the optimal opportunity maintenance strategy for equipment from the point of systemic view.

\section{Conclusion}

Based on the equipment condition-based maintenance, in this paper, a mathematical model of the system conditionbased maintenance decision considering the opportunistic maintenance strategy is proposed. The results on three simple substations and IEEE-RTS79 system verify that the effectiveness of the proposed method and the major contributions of this paper are summarized as follows:

(1) It is necessary to consider opportunistic maintenance in the process of equipment condition-based maintenance decision-making, which is conducive to coordinate the relationship between equipment maintenance and system operation and improve the reliability and economy of the system operation.

(2) Compared with the existing researches, the proposed model can flexibly choose the opportunistic maintenance strategies according to the actual condition of the system equipment and system risk.

However, the CBM decision-making of power system is a complex combinatorial optimization problem; with the increase of the system scale, the solution efficiency will decrease. How to improve the existing intelligent search algorithm to increase the solution efficiency is the focus of our future research.

\section{Data Availability}

The data, provided in the article, were collected from the National Power Grid Corp and China Electric Power Press. They are available in the public domain without restriction.

\section{Conflicts of Interest}

The authors declare that there are no conflicts of interest.

\section{Acknowledgments}

This work was supported by 2020 Science and Technology Projects in Qingdao West Coast New District (Source Innovation Project) (no. 2020-92).

\section{References}

[1] Y. Tan, F. Qiu, A. K. Das, D. S. Kirschen, P. Arabshahi, and J. Wang, "Scheduling post-disaster repairs in electricity distribution networks," IEEE Transactions on Power Systems, vol. 34, no. 4, pp. 2611-2621, 2019.

[2] M. Compare and E. Zio, "Genetic algorithms in the framework of Dempster-Shafer theory of evidence for maintenance optimization problems," IEEE Transactions on Reliability, vol. 64, no. 2, pp. 645-660, 2015.

[3] R. Gong, S. Li, and W. Peng, "Research on multi-attribute decision-making in condition-based maintenance for power transformers based on cloud and Kernel vector space models," Energies, vol. 13, no. 22, p. 5948, 2020.

[4] Y. Chen, W. Gong, D. Xu, and R. Kang, "Imperfect maintenance policy considering positive and negative effects for deteriorating systems with variation of operating conditions," IEEE Transactions on Automation Science and Engineering, vol. 15, no. 2, pp. 872-878, 2018.

[5] X. Zhang and J. Zeng, "A general modeling method for opportunistic maintenance modeling of multi-unit systems," Reliability Engineering \& System Safety, vol. 140, pp. 176-190, 2015.

[6] Y. Shi, W. Zhu, Y. Xiang, and Q. Feng, "Condition-based maintenance optimization for multi-component systems subject to a system reliability requirement," Reliability Engineering \& System Safety, vol. 202, Article ID 107042, 2020.

[7] J. Huang, Q. Chang, J. Zou, and J. Arinez, "A real-time maintenance policy for multi-stage manufacturing systems considering imperfect maintenance effects," IEEE Access, vol. 6, pp. 62174-62183, 2018.

[8] I. B. Gertsbakh, "Optimal group preventive maintenance of a system with observable state parameter," Advances in Applied Probability, vol. 16, no. 4, pp. 923-925, 1984.

[9] S.-H. Sheu and J.-P. Jhang, "A generalized group maintenance policy," European Journal of Operational Research, vol. 96, no. 2, pp. 232-247, 1997.

[10] J. S. Dagpunar, "A maintenance model with opportunities and interrupt replacement options," Journal of the Operational Research Society, vol. 47, no. 11, pp. 1406-1409, 1996.

[11] F. Camci, "System maintenance scheduling with prognostics information using genetic algorithm," IEEE Transactions on Reliability, vol. 58, no. 3, pp. 539-552, 2009.

[12] S. Lin, R. Fan, D. Feng, C. Yang, Q. Wang, and S. Gao, "Condition-based maintenance for traction power supply equipment based on partially observable markov decision process," IEEE Transactions on Intelligent Transportation Systems, pp. 1-15, 2020.

[13] M. S. Alvarez-Alvarado and D. Jayaweera, "Reliability-based smart-maintenance model for power system generators," IET Generation, Transmission and Distribution, vol. 14, no. 9, pp. 1770-1780, 2020.

[14] S. Lakshminarayanan and D. Kaur, "Optimal maintenance scheduling of generator units using discrete integer cuckoo search optimization algorithm," Swarm and Evolutionary Computation, vol. 42, pp. 89-98, 2018.

[15] Y. He, F. Liu, J. Cui et al., "Reliability-oriented design of integrated model of preventive maintenance and quality control policy with time-between-events control chart," Computers \& Industrial Engineering, vol. 129, pp. 228-238, 2019.

[16] Y. Zhao, Y. He, D. Zhou et al., "Functional risk-oriented integrated preventive maintenance considering product quality loss for multistate manufacturing systems," International Journal of Production Research, vol. 59, no. 4, pp. 1003-1020, 2021.

[17] Z. Zhao, B. Xiao, N. Wang, X. Yan, and L. Ma, "Selective maintenance optimization for a multi-state system with degradation interaction," IEEE Access, vol. 7, pp. 99191-99206, 2019.

[18] C. Zhang, W. Gao, T. Yang, and S. Guo, "Opportunistic maintenance strategy for wind turbines considering weather 
conditions and spare parts inventory management," Renewable Energy, vol. 133, pp. 703-711, 2019.

[19] M. Urbani, M. Brunelli, and M. Collan, "A comparison of maintenance policies for multi-component systems through discrete event simulation of faults," IEEE Access, vol. 8, pp. 143654-143664, 2020.

[20] K. T. P. Nguyen and K. Medjaher, "A new dynamic predictive maintenance framework using deep learning for failure prognostics," Reliability Engineering \& System Safety, vol. 188, pp. 251-262, 2019.

[21] M. C. A. Olde Keizer, S. D. P. Flapper, and R. H. Teunter, "Condition-based maintenance policies for systems with multiple dependent components: a review," European Journal of Operational Research, vol. 261, no. 2, pp. 405-420, 2017.

[22] M. Yildirim, N. Z. Gebraeel, and X. A. Sun, "Integrated predictive analytics and optimization for opportunistic maintenance and operations in wind farms," IEEE Transactions on Power Systems, vol. 32, no. 6, pp. 4319-4328, 2017.

[23] P. Zhou and P. T. Yin, "An opportunistic condition-based maintenance strategy for offshore wind farm based on predictive analytics," Renewable and Sustainable Energy Reviews, vol. 109, pp. 1-9, 2019.

[24] Y. Wang, E. Elahi, and L. Xu, "Selective maintenance optimization modelling for multi-state deterioration systems considering imperfect maintenance," IEEE Access, vol. 7, pp. 62759-62768, 2019.

[25] Y. Liu, Y. Su, Y. Xiang, J. Liu, L. Wang, and W. Xu, “Operational reliability assessment for gas-electric integrated distribution feeders," IEEE Transactions on Smart Grid, vol. 10, no. 1, pp. 1091-1100, 2018.

[26] A. D. Orcesi and D. M. Frangopol, "Probability-based multiple-criteria optimization of bridge maintenance using monitoring and expected error in the decision process," Structural and Multidisciplinary Optimization, vol. 44, no. 1, pp. 137-148, 2011.

[27] Z. Chen, Y. He, Y. Zhao et al., "Mission reliability-oriented selective maintenance optimization for intelligent multistate manufacturing systems with uncertain maintenance quality," IEEE Access, vol. 7, pp. 109804-109816, 2019.

[28] Y. He, Z. Chen, Y. Zhao, X. Han, and D. Zhou, "Mission reliability evaluation for fuzzy multistate manufacturing system based on an extended stochastic flow network," IEEE Transactions on Reliability, vol. 69, no. 4, pp. 1239-1253, 2020.

[29] Q. Huang, S. Jing, J. Li et al., "Smart substation: state of the art and future development," IEEE Transactions on Power Delivery, vol. 32, no. 2, pp. 1098-1105, 2016.

[30] Y. Zhang, X. Ji, Y. Yu, B. Xu, X. Han, and L. Zhang, "Maintenance optimization for combined heat and power unit based on opportunistic maintenance strategy," in Proceedings of the 2020 IEEE/IAS Industrial and Commercial Power System Asia (IङCPS Asia), pp. 519-526, Weihai, China, 2020.

[31] P. Brémaud, Probability Theory and Stochastic Processes, Springer, Berlin, Germany, 2020.

[32] W. Li, Risk Assessment of Power Systems: Models, Methods, and Applications, John Wiley \& Sons, Hoboken, NJ, USA, 2014.

[33] B. Mor, S. Garhwal, and A. Kumar, "A systematic review of hidden Markov models and their applications," Archives of Computational Methods in Engineering, vol. 28, pp. 14291448, 2020.

[34] E. A. Elsayed, Reliability Engineering, John Wiley \& Sons, Hoboken, NJ, USA, 2020.

[35] Z. Wu, B. Guo, Axita, X. Tian, and L. Zhang, "A dynamic condition-based maintenance model using inverse Gaussian process," IEEE Access, vol. 8, pp. 104-117, 2020.
[36] T. Wu, L. Yang, X. Ma, Z. Zhang, and Y. Zhao, "Dynamic maintenance strategy with iteratively updated group information," Reliability Engineering \& System Safety, vol. 197, Article ID 106820, 2020. 